\title{
Refractory Dedifferentiated Liposarcoma
}

National Cancer Institute

\section{Source}

National Cancer Institute. Refractory Dedifferentiated Liposarcoma. NCI Thesaurus.

Code C150593.

Dedifferentiated liposarcoma that does not respond to treatment. 